\title{
Leishmania mortality in sand fly blood meal is not species-specific and does not result from direct effect of proteinases
}

\author{
Katerina Pruzinova ${ }^{1 *}, J^{\prime}$ ovana Sadlova', Jitka Myskova ${ }^{1}$, Tereza Lestinova ${ }^{1}$, Jozef Janda ${ }^{2}$ and Petr Volf ${ }^{1}$
}

\begin{abstract}
Background: Leishmania development in sand flies is confined to the alimentary tract and is closely connected with blood meal digestion. Previously, it has been published that activities of sand fly midgut proteases are harmful to Leishmania, especially to amastigote-promastigote transition forms. However, our experiments with various Leishmania-sand fly pairs gave quite opposite results.

Methods: We evaluated the effect of semi-digested midgut content on different life stages of Leishmania donovani and Leishmania major in vitro. Various morphological forms of parasites, including macrophage-derived amastigotes and transition forms, were incubated $2 \mathrm{~h}$ with midguts dissected at various intervals (6-72 h) post-blood meal or with commercially available proteinase, and their viability was determined using flow cytometry. In parallel, using amastigote-initiated experimental infections, we compared development of L. donovani in sand flies that are either susceptible (Phlebotomus argentipes and P. orientalis) or refractory (P. papatasi and Sergentomyia schwetzi) to this parasite.

Results: In vitro, sand fly midgut homogenates affected L. major and L. donovani in a similar way; in all sand fly species, the most significant mortality effect was observed by the end of the blood meal digestion process. Surprisingly, the most susceptible Leishmania stages were promastigotes, while mortality of transforming parasites and amastigotes was significantly lower. Parasites were also susceptible to killing by rabbit blood in combination with proteinase, but resistant to proteinase itself. In vivo, L. donovani developed late-stage infections in both natural vectors; in $P$. argentipes the development was much faster than in P. orientalis. On the other hand, in refractory species $P$. papatasi and $S$. schwetzi, promastigotes survived activity of digestive enzymes but were lost during defecation.

Conclusions: We demonstrated that Leishmania transition forms are more resistant to the killing effect of semi-digested blood meal than 24 h-old promastigotes. Data suggest that Leishmania mortality is not caused directly by sand fly proteases, we assume that this mortality results from toxic products of blood meal digestion. Survival of L. donovani promastigotes in refractory sand flies until blood meal defecation, together with similar mortality of Leishmania parasites incubated in vitro with midgut homogenates of susceptible as well as refractory species, contradict the previously raised hypotheses about the role of midgut proteases in sand fly vector competence to Leishmania.
\end{abstract}

Keywords: Sand fly, Phlebotomus, Sergentomyia, Leishmania donovani, Leishmania major, Blood meal digestion, Proteases

\footnotetext{
*Correspondence: katerina.pruzinova@gmail.com

'Department of Parasitology, Faculty of Science, Charles University, Prague,

Czech Republic

Full list of author information is available at the end of the article
} 


\section{Background}

Protozoan parasites of the genus Leishmania (Kinetoplastida: Trypanosomatidae) have a digenetic life-cycle consisting of extracellular promastigotes developing in sand fly vectors (Diptera: Phlebotominae) and intracellular amastigotes in mammalian hosts [1, 2]. Leishmania donovani causes human visceral leishmaniasis (VL, kalaazar), which is a deadly disease occurring mainly in the Indian subcontinent and East Africa. The most important vectors of this parasite are Phlebotomus argentipes and $P$. orientalis $[3,4]$. Leishmania major, which is mainly transmitted by $P$. papatasi and $P$. duboscqi, is the causative agent of cutaneous leishmaniasis $(\mathrm{CL})$, and is widely distributed in arid and savannah areas of subSaharan and North Africa, Middle East and Indian subcontinent $[4,5]$.

Various sand fly species differ in vector competence to various Leishmania species, and the knowledge of factors affecting vector competence is crucial from an epidemiological point of view $[2,6]$. Leishmania development in sand flies is confined to the alimentary tract and is closely connected with blood meal digestion $[1,2]$. As the majority of nutrients in the ingested blood is constituted by protein molecules, the proteolytic enzymes play the main role during blood meal digestion of sand flies, most of them being serine proteases, namely trypsin- and chymotrypsin-like molecules [7-9]. The midgut epithelial cells start to produce trypsin- and chymotrypsin-like proteases after the engorgement of the blood meal, activity levels are significantly increased from $6 \mathrm{~h}$ post-blood meal (pbm) and peak at 12-48 h $\mathrm{pbm}$, depending on sand fly species [10-13].

It has been published repeatedly that proteolytic activities in the sand fly midgut affect Leishmania development [14-16]. According to Schlein \& Romano [17] and Borovsky \& Schlein [14], the specific components of trypsin-like activity cause the reduction of parasite numbers, or even death, of $L$. donovani promastigotes in the "non-compatible" vector $P$. papatasi, whereas the ability to influence this factor allows L. major to survive and develop within its natural vector. Using experimental infections of sand flies by promastigotes and the exogenous suppression of midgut proteolytic activity by soybean trypsin inhibitor, same authors $[14,16]$ established the hypothesis that activities of midgut proteases influence the vector competence of sand flies. Moreover, Pimenta et al. [15] have shown that the susceptibility of Leishmania parasites to destruction by midgut proteolytic activity in the natural vector is stage-specific. In their in vitro experiments, amastigotes and fullytransformed promastigotes of L. major were relatively resistant to $P$. papatasi proteases, while the parasites within amastigote-promastigote transition were highly susceptible to be killed.
In the present study, we evaluated the killing effects of midgut homogenates of various sand fly species on different morphological forms of $L$. donovani and L. major. Then, we use the most susceptible form, promastigotes, to study dynamics of this killing activity in four different sand fly species differing in susceptibility to $L$. donovani: two natural vectors, $P$. argentipes and $P$. orientalis $[4,12,18]$, and two refractory ones, $P$. papatasi and Sergentomyia schwetzi [14, 16, 19, 20]. In parallel experiments in vivo, we compared $L$. donovani development in four sand fly species mentioned above, focussing on timing of parasite loss in refractory species.

\section{Methods}

\section{Sand flies}

Colonies of $P$. argentipes, $P$. orientalis, $P$. papatasi and $S$. schwetzi were maintained under standard conditions as described previously [21]. Three to seven day old females were fed on anesthetized mice, maintained at $26{ }^{\circ} \mathrm{C}$ and their midguts were dissected 6, 24, 32, 48 and $72 \mathrm{~h} \mathrm{pbm}$.

\section{Leishmania parasites and macrophages}

Leishmania donovani promastigotes transfected with green fluorescence protein-GFP (MHOM/ET/2010/DM1033/GR374) or red fluorescence protein-RFP (MHOM/ ET/2009/AM459) and L. major promastigotes expressing dsRed fluorescence protein (MHOM/IR/-/173) were maintained at $23{ }^{\circ} \mathrm{C}$ in Medium 199 (Sigma-Aldrich, St. Louis, USA), supplemented with $10 \%$ foetal calf serum (Thermo Fisher Scientific, Waltham, USA), 1\% BME vitamins (Sigma-Aldrich), 2\% human urine and $250 \mu \mathrm{g} / \mathrm{ml}$ amikacin (Amikin, Medopharm, Prague, Czech Republic). Leishmania donovani promastigotes transfected with green and red fluorescence protein were maintained under pressure of selective antibiotic G $418(150 \mu \mathrm{g} / \mathrm{ml}$; Sigma-Aldrich) and hygromycin B (150 $\mathrm{\mu g} / \mathrm{ml}$; Sigma-Aldrich), respectively.

Amastigote stages of $L$. donovani were grown in $\mathrm{J} 744$ macrophage cell lines originating from $\mathrm{BALB} / \mathrm{c}$ mice. Amastigotes of $L$. major were grown in bone-marrow macrophages (BMMs) differentiated from precursor cells of BALB/c mice in the presence of L929 fibroblast cell culture supernatant as a source of macrophage colony stimulating factor (M-CSF). Macrophages were exposed to stationary-phase promastigotes at a parasite to macrophage ratio of 8 promastigotes to 1 macrophage in 24well plates $(1 \mathrm{ml} /$ well $)$. Both infected and uninfected macrophages were cultured in complete RPMI-1640 medium (Sigma-Aldrich), containing 10\% FBS, 1\% penicillin-streptomycin (Sigma-Aldrich), $2 \mathrm{mM}$ of Lglutamine (Sigma-Aldrich) and $0.05 \mathrm{mM}$ of $\beta$-mercaptoethanol at $37{ }^{\circ} \mathrm{C}$ with $5 \% \mathrm{CO}_{2}$.

We used macrophage-derived amastigotes in order to keep the 3R rules (refinement-replacement-reduction) 
and decrease the number of animals used in experiments. In comparison with tissue amastigotes, those isolated from the in vitro culture system were shown to be similarly infective to laboratory animals [22].

\section{Promastigote transformation assay}

After 72-96 h post-infection, infected macrophages were disrupted by $0.016 \%$ SDS (Sigma-Aldrich) for 5-10 min and lysed cells were scraped by plunger from $1 \mathrm{ml}$ insulin syringe. Leishmania donovani or major amastigotes were released to the Medium 199 and were allowed to transform at $23{ }^{\circ} \mathrm{C}$. Three different Leishmania stages were used for in vitro experiments: amastigotes (immediately released from macrophages), parasites within amastigote-promastigote transition ( $5 \mathrm{~h}$ after the release from macrophages) and promastigote-like form (24 h after the release from macrophages). Determination of Leishmania stages was established on the basis of observation under the microscope; on $0 \mathrm{~h}$ all parasites were amastigotes, on $5 \mathrm{~h}$ there were transient forms with very small flagella and on $24 \mathrm{~h} 100 \%$ of parasites in culture were promastigotes. This timing of parasite transformation was similar to Pimenta et al. [15], who used tissue amastigotes.

\section{Incubation of Leishmania with sand fly midgut homogenates and blood derivatives}

Sand fly females were fed on anesthetized mice and dissected at different times post-blood meal (pbm). Dissection of midguts was semi-sterile and performed on ice, the preparation of one sample took maximum $30 \mathrm{~min}$ and the samples were immediately placed in a freezer $\left(-80{ }^{\circ} \mathrm{C}\right)$. In experiments, midguts were homogenised while defrosting, centrifuged $(12,000 \times g)$ and the supernatant was incubated with parasites.

In the first series of experiments, various $L$. donovani (GFP) or L. major (dsRed) stages were incubated with $P$. argentipes, $P$. orientalis, $P$. papatasi or S. schwetzi midgut homogenates (dissected $24 \mathrm{~h} \mathrm{pbm}$ ) for $2 \mathrm{~h}$ in a microwell plate (1000 Leishmania/midgut). In the second series of the experiments, L. donovani (GFP) promastigotes (24 $\mathrm{h}$ after the release from macrophages) were incubated with $P$. argentipes, $P$. orientalis, $P$. papatasi or S. schwetzi midgut homogenates (dissected 6, 24, 32,48 and $72 \mathrm{~h} \mathrm{pbm}$ ) for $2 \mathrm{~h}$ in a microwell plate (1000 Leishmania/midgut). In addition, promastigotes $24 \mathrm{~h}$ post-transition were incubated with proteinase $\mathrm{K}$ (6.7 $\mathrm{mg} / \mathrm{ml}, 2 \mathrm{U} / \mathrm{mg}$ protein; Roche, Mannheim, Germany), rabbit blood, red cells of rabbit blood, human haemoglobin (220 mg/ml; Sigma-Aldrich), rabbit blood + proteinase $\mathrm{K}$, red cells + proteinase $\mathrm{K}$ and haemoglobin + proteinase K. Leishmania parasites incubated for $2 \mathrm{~h}$ with saline instead of midgut homogenates were used as a negative control and parasites killed by $1 \%$ formaldehyde and permeabilised by $0.5 \%$ Triton X-100 (Sigma-Aldrich) were used as a positive control.

After the incubation, parasites with midgut homogenate were transferred to saline solution, dead cell were marked with DAPI (4',6-Diamidine-2'-phenylindole dihydrochloride, $0.005 \mathrm{mg} / \mathrm{ml}$; Thermo Fisher Scientific) and analysed by flow cytometry. Flow cytometry measurements were performed using flow cytometer CytoFLEX S (Beckman Coulter, Inc., Brea, California, USA) equipped with 4 lasers $(405 \mathrm{~nm}, 488 \mathrm{~nm}, 561 \mathrm{~nm}$ and $638 \mathrm{~nm}$ ) and 13 fluorescence detectors. GFP was excited using $488 \mathrm{~nm}$ laser and its fluorescence emission was detected using 525/40 filter, dsRed was excited using $561 \mathrm{~nm}$ laser and its fluorescence emission was detected using $585 / 42$ bandpass filter, DAPI was excited by $405 \mathrm{~nm}$ laser and detected using 450/50 filter. Analysis of cytometry data was performed using CytExpert software (Beckman Coulter).

The experiments were conducted in duplets and repeated twice. Statistical evaluations were performed by the ANOVA and post-hoc Tukey HSD test using SPSS Statistics 23.0 software.

\section{Sand fly infections}

For amastigote-initiated infections, L. donovani (RFP) were co-cultivated with mouse macrophage line J774 for $72 \mathrm{~h}$ and non-internalized parasites were removed by thorough washing with pre-heated culture medium. Numbers of amastigotes per macrophages were established by fluorescent microscopy. The macrophages were removed from the culture plates by extensive washing with cold saline, centrifuged at $300 \times \mathrm{g}, 4{ }^{\circ} \mathrm{C}$ for $10 \mathrm{~min}$ and resuspended in heat-inactivated rabbit blood for sand fly infections at the concentration of $1 \times 10^{6}$ amastigotes $/ \mathrm{ml}$.

Females were dissected at several time intervals postblood meal (pbm) and the abundance and location of Leishmania infections in the sand fly digestive tract were examined by fluorescent microscopy. Parasite loads were graded according to Myskova et al. [23] as light $(<100$ parasites per gut), moderate (100-1000 parasites per gut) and heavy (> 1000 parasites per gut). Experiments with each Leishmania-sand fly combination were repeated four to six times.

\section{Morphometry of parasites}

Midgut smears of sand flies infected with Leishmania parasites were fixed with methanol, stained with Giemsa, examined under an Olympus BX51 light microscope and photographed with an Olympus D70 camera. Body length, flagellar length and body width of parasites were measured using Image-J software. Four morphological forms were distinguished, as described in Sadlova et al. [24]: procyclic promastigotes (PP), elongated nectomonads (EN), metacyclic promastigotes (MP) and short 
promastigotes (SP). Haptomonads were not distinguished as representation of these attached forms is in principle underestimated on gut smears. In total, 160 randomly selected promastigotes from four females/ smears for each sand fly species and time pbm were measured for comparison of amastigote-initiated $L$. donovani infections in four sand fly species.

\section{Results}

Effects of sand fly midgut homogenates on various forms of $L$. donovani and L. major

First we tested the killing effect of midgut homogenates from four sand fly species dissected $24 \mathrm{~h}$ pbm. All parasite forms tested (amastigotes, transition forms and promastigotes) of both Leishmania species were relatively resistant to killing effect of midgut homogenates of $P$. orientalis, $P$. papatasi and S. schwetzi; the mortality ranged between 1 and $8 \%$ in $L$. donovani (Fig. 1), 5-30\% in L. major (Fig. 2) and was comparable to negative controls. Conversely, significant killing effect was caused by midgut homogenates of $P$. argentipes; the most susceptible forms were promastigotes with mortality about $60 \%$ (L. donovani) and 78\% (L. major), mortality of transition forms and amastigotes was significantly lower, 28-37\% and $17-22 \%$, respectively (Tukey HSD, $P<0.001$; Figs. 1 and 2). Representative images of flow cytometry analysis are shown in Additional file 1: Figures S1-S7.

\section{Effect of midgut homogenates from different sand fly species on $L$. donovani promastigotes}

The most susceptible stages, promastigotes (24 h after the release from the macrophages) were tested with sand fly midgut homogenates dissected at different times pbm (6, 24, 32, 48 and 72 h). Results are shown in Fig. 3a. Significant differences found in killing effect of various homogenates and time intervals (ANOVA, $\left.\quad F_{(6,27)}=834, \quad P<0.0001\right)$ correlated with differences in the time course of blood meal digestion: Phlebotomus argentipes digest fast and, therefore, significant promastigote mortality (Tukey HSD, $P<0.0001$ ) was observed in groups incubated with homogenates obtained at $24 \mathrm{~h}$ pbm (mortality $55 \%$ ), or 32 and $48 \mathrm{~h} \mathrm{pbm} \mathrm{(mortality} \mathrm{100 \% ).} \mathrm{In} \mathrm{con-}$ trast, $P$. orientalis digest slowly and significantly increased killing effect (Tukey HSD, $P<0.0001$ ) was observed for homogenates dissected $72 \mathrm{~h}$ pbm (mortality 50\%,). For P. papatasi and S. schwetzi, significantly enhanced mortality (Tukey HSD: $P<0.0001$ ) was observed in promastigotes incubated with midguts dissected 32, 48 and $72 \mathrm{~h} \mathrm{pbm}$. As a negative control, we used midguts dissected $6 \mathrm{~h}$ pbm which did not cause any mortality of parasites in any of sand fly species (Fig. 3a).

In addition, we tested the effect of proteinase $\mathrm{K}$ and blood or blood derivatives on promastigotes. The results are shown in Fig. 3b. Proteinase $\mathrm{K}$ itself had no effect on parasites (mortality 1\%), blood partly killed Leishmania (mortality 10\%) while blood with addition of proteinase $\mathrm{K}$ caused significantly higher mortality (60\%,Tukey HSD, $P<0.0001)$. A similar enhancing effect was observed in red cells, which killed about $30 \%$ of parasites, while red cells in combination with proteinase $\mathrm{K}$ caused significantly higher mortality of parasites $(90 \%$, Tukey HSD, $P<0.0001)$. Haemoglobin itself killed about $70 \%$ of Leishmania and haemoglobin with proteinase $\mathrm{K}$ caused mortality about $85 \%$; this difference was not significant (Tukey HSD, $P=0.903$ ).

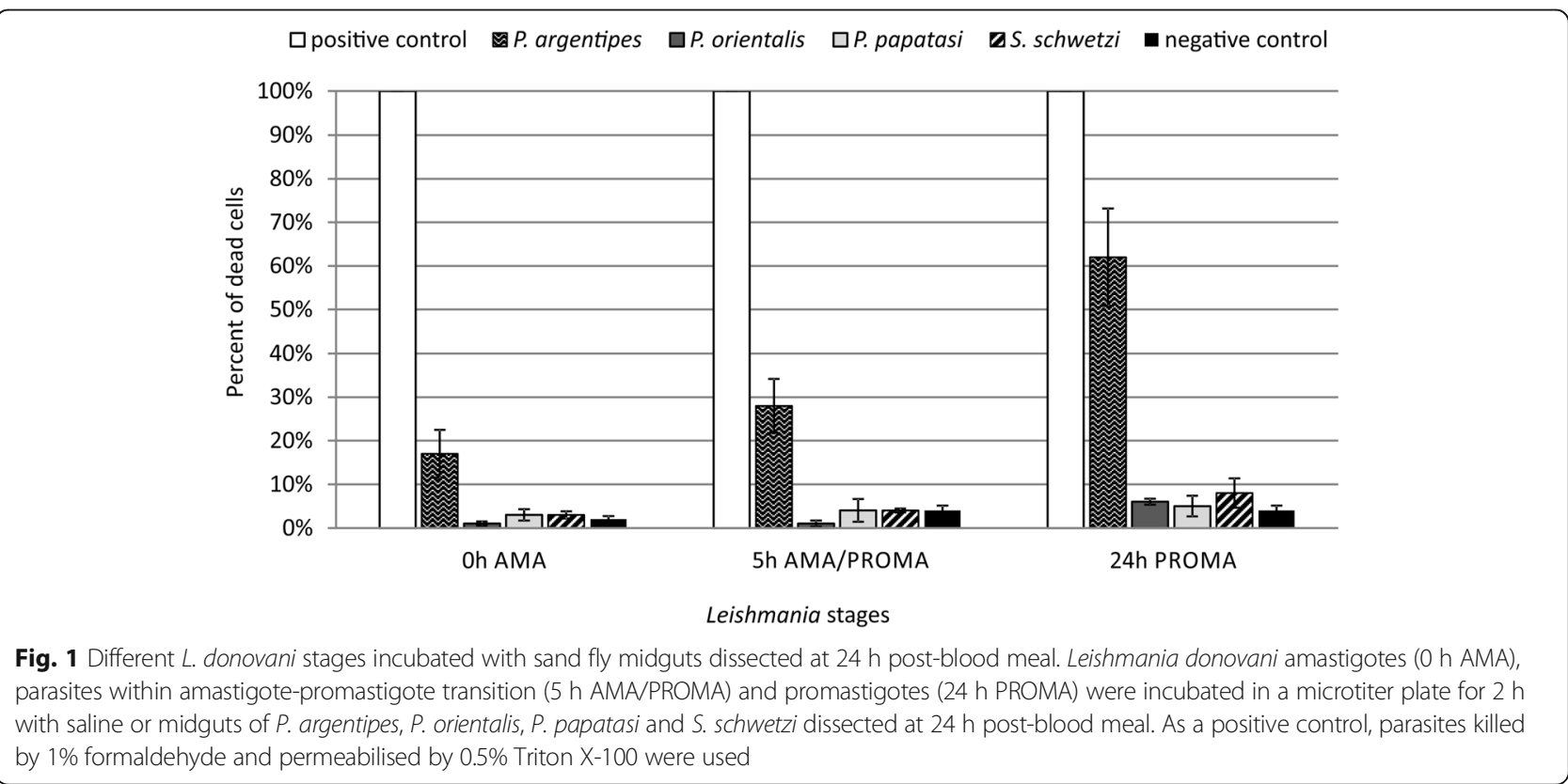




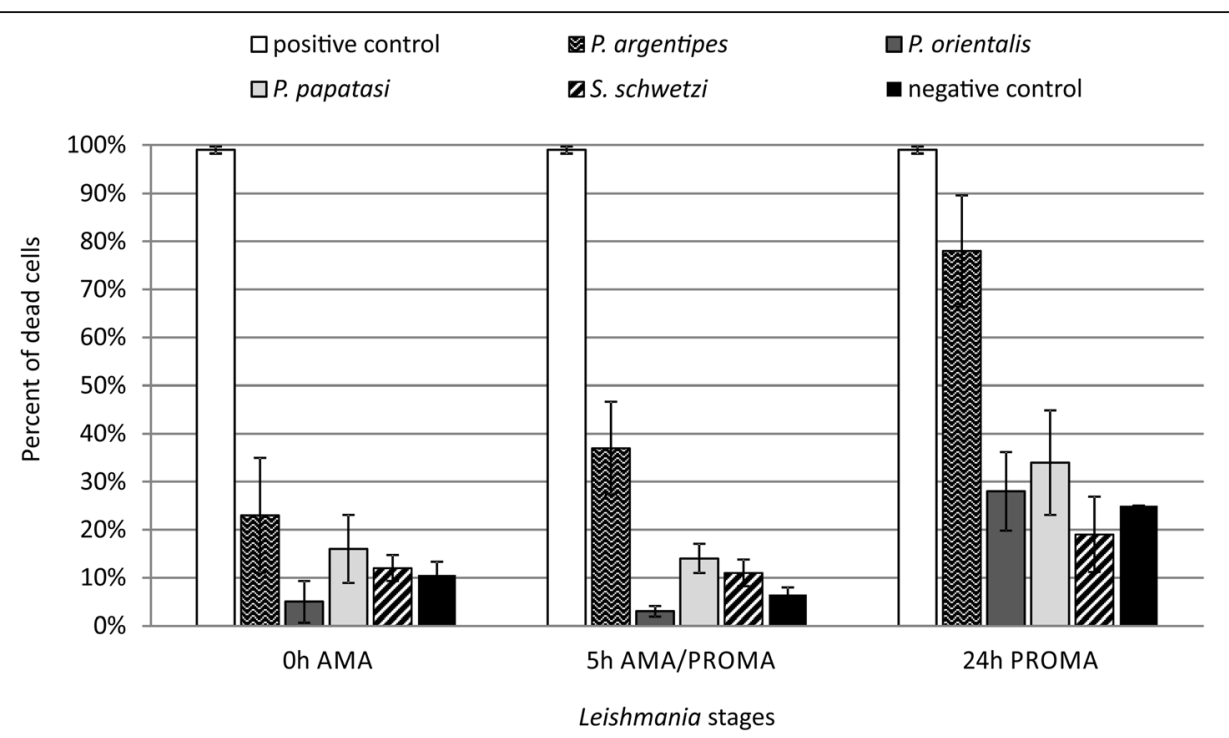

Fig. 2 Different L. major stages incubated with sand fly midguts dissected at $24 \mathrm{~h}$ post-blood meal. Leishmania major amastigotes (0 h AMA), parasites within amastigote-promastigote transition ( $5 \mathrm{~h}$ AMA/PROMA) and promastigotes ( $24 \mathrm{~h}$ PROMA) were incubated in a microtiter plate for $2 \mathrm{~h}$ with saline or midguts of P. argentipes, P. orientalis, P. papatasi and S. schwetzi dissected at $24 \mathrm{~h}$ post-blood meal. As a positive control, parasites killed by $1 \%$ formaldehyde and permeabilised by $0.5 \%$ Triton $X-100$ were used

\section{Comparison of L. donovani development in four vector species}

The development of $L$. donovani was compared in detail in two natural vectors ( $P$. argentipes and $P$. orientalis) and two refractory species (P. papatasi and S. schwetzi), using amastigote-initiated experimental infections of sand flies.

Before defecation, by day $2 \mathrm{pbm}$, the infection rates were similar in all four sand fly species: more than $80 \%$ of females were infected and heavy or moderate intensities of infections prevailed (Fig. 4a). However, the location of parasites differed among sand fly species. In all $P$. papatasi and $S$. schwetzi, as well as in $93 \%$ of $P$. orientalis, infections were still enclosed inside the peritrophic matrix (PM). Conversely, in $P$. argentipes, most infections reached the abdominal midgut (AMG) or even the thoracic midgut (TMG) (Fig. 4b). Faster development of L. donovani in $P$. argentipes was reflected by higher proportion of elongated nectomonads in comparison with prevailing procyclic forms in other three sand fly species (Fig. 4c).

By day 3 pbm, the pattern of Leishmania development highly differed among sand fly species studied. In $P$. orientalis, both rates and intensities of infections were very high and parasites were found in a wide range of locations, either still remaining with digested blood in the endoperitrophic space $(11 \%)$ or reaching the AMG (26\%) or the TMG (55\%) or even the cardia region (8\%). In $P$. argentipes, $83 \%$ of females already defecated blood remnants and most parasites reached the TMG (57\%) or even the cardia region (39\%). Importantly, in $P$. papatasi, no parasites were found in females which already defecated (19\%). In non-defecated females, living parasites were either enclosed inside the endoperitrophic space $(45 \%)$ or reached the AMG (55\%). Similarly, in $S$. schwetzi, defecated females were parasite-free (66\%), in non-defecated individuals, parasites were enclosed inside the PM. While elongated nectomonads prevailed in proven vectors and metacyclic forms were also already present, procyclic promastigotes remained the prevailing form in both refractory species (Fig. 4c).

Late-phase infections differed considerably among the four sand fly species. In $P$. orientalis and P. argentipes (proven vectors of $L$. donovani), parasites developed heavy infections, infection rates did not fall below $80 \%$ and colonization of the stomodeal valve (SV) was observed in $59 \%$ and $44 \%$ of infected females by day 6 pbm, and $77 \%$ and $72 \%$ by day 10 PBM, respectively (Fig. 4a, b). Proportion of metacyclic promastigotes did not differ significantly between $P$. orientalis and $P$. argentipes (Fig. 4c). On the other hand, infection rates in P. papatasi rapidly decreased to $5 \%$ and $2 \%$ by day 6 and $10 \mathrm{pbm}$, respectively, and in those few positive females promastigotes never colonized the SV. No Leishmania infection was detected in S. schwetzi after defecation.

\section{Discussion}

More than two decades ago it was proposed that (i) activities of sand fly midgut proteases are harmful to Leishmania in non-compatible vectors [14, 16, 17], and (ii) in natural vectors, the most susceptible stages to digestive enzymes are forms in transition from amastigotes 


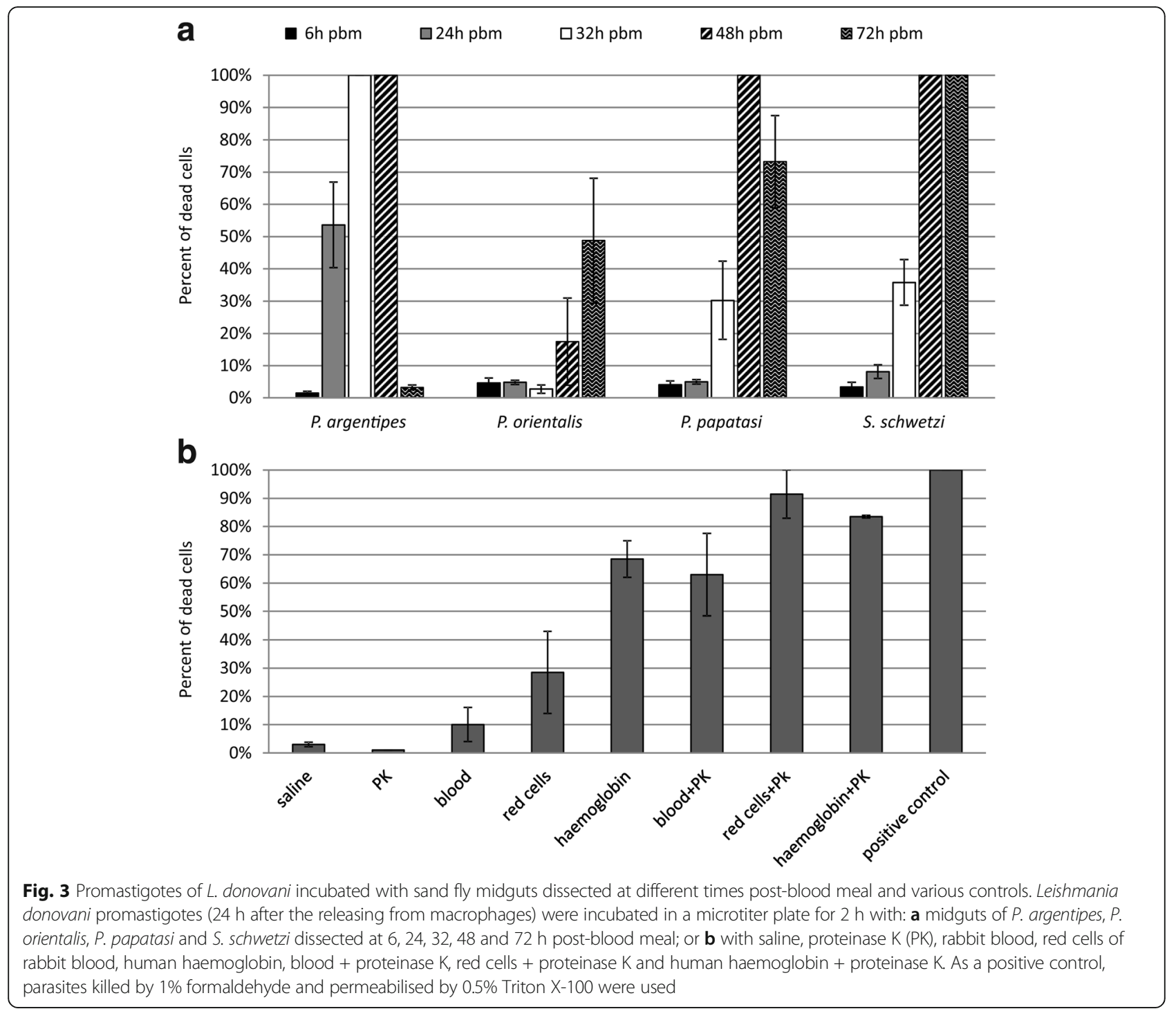

to promastigotes [15]. In the present study, we tested both these hypotheses and evaluated the effect of sand fly midgut digestion on various $L$. donovani and $L$. major stages in vivo as well as in vitro.

Interestingly, for both parasite species tested, $L$. donovani and L. major, promastigotes were found to be the most susceptible forms to killing by midgut homogenates in vitro, while mortalities of transition forms and amastigotes were significantly lower. These findings are in contrast with the study of Pimenta et al. [15], where authors reported 95\% reduction in numbers of viable transforming parasites, but fresh amastigotes and $18 \mathrm{~h}$ old promastigotes were less susceptible to be killed.

Incubation of $L$. donovani promastigotes with midgut homogenates dissected from various sand fly species at different times pbm supported the fact that effect of blood digestion on parasites is not selective and speciesspecific. The mortality of parasites was more influenced by the time pbm of used midgut homogenates than by the sand fly species. The four sand fly species substantially differ in kinetics of blood meal digestion [12], which was well reflected in time-course of the harmful effect of midgut homogenates on $L$. donovani in these experiments. In susceptible as well as refractory parasite-vector combinations, the mortality of parasites increased with the degree of digested blood and the highest mortality effect was observed at the end of their blood meal digestion.

Experiments in vitro also showed susceptibility of Leishmania parasites to human haemoglobin or combination of rabbit blood or red blood cells with proteinase, but demonstrated their resistance to proteinase itself. As haemoglobin negatively influences the development of infective Leishmania promastigotes in culture [25], and haem generated after haemoglobin hydrolysis is well known for its cytotoxicity [26], we assume that 


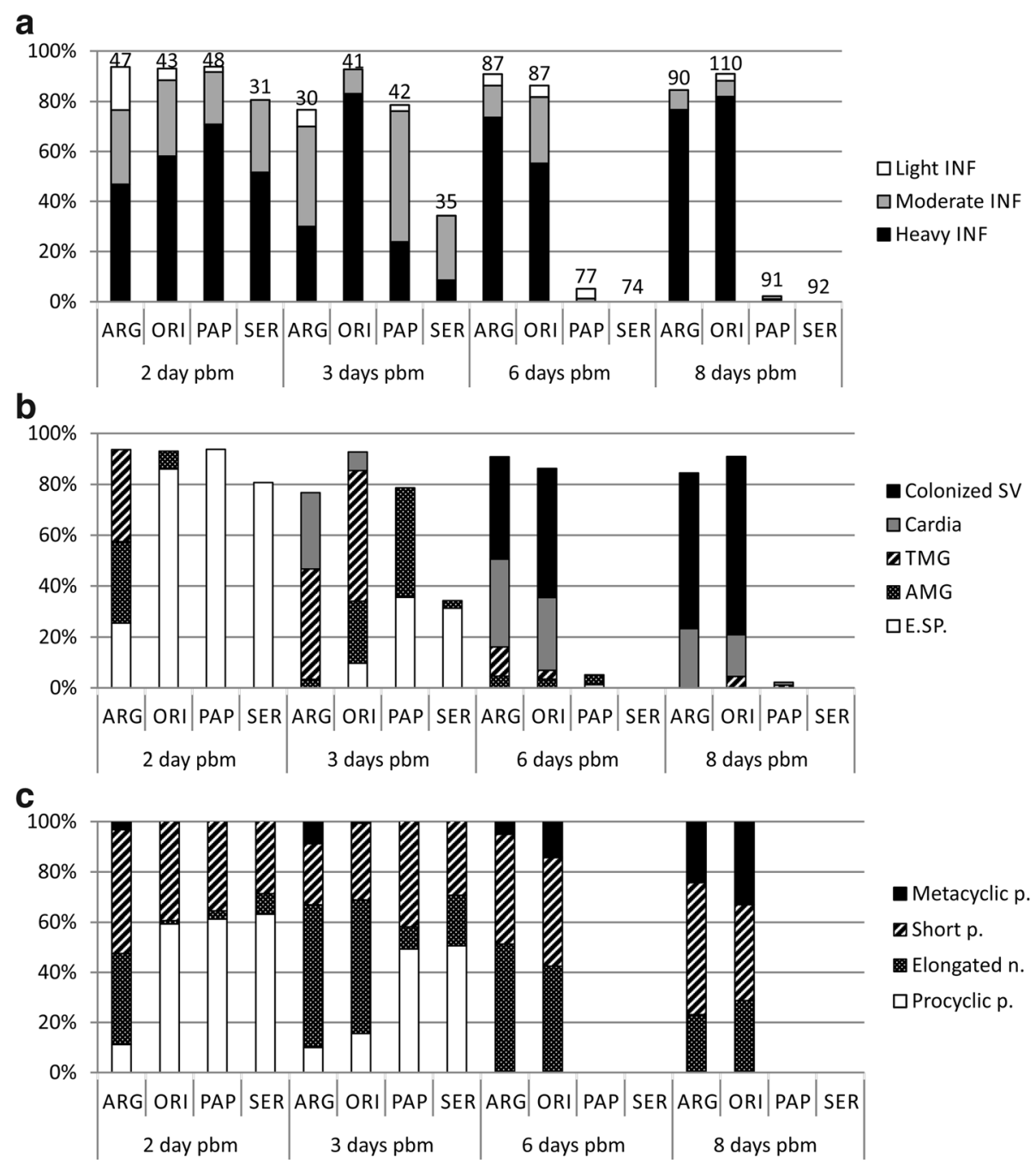

Fig. 4 Amastigote-initiated infections of L. donovani in four sand fly species: P. orientalis (ORI), P. argentipes (ARG), P. papatasi (PAP) and S. schwetzi (SER). a Rates and intensities of $L$. donovani infections. Numbers of dissected females are shown above bars. Differences between species were evaluated using Chi-square test: day 2 PBM, $X^{2}=20.009, d f=9, P=0.018$; day 3 pbm, $X^{2}=74.880, d f=9, P<0.0001 ;$ day 6 pbm, $X^{2}=255.6, d f=9$, $P<0.0001$; day $10 \mathrm{pbm}, X^{2}=296.2, d f=9, P<0.0001$. b Location of $L$. donovani in infected sand flies. Differences between species were evaluated using Chi-square test: day 2 PBM, $x^{2}=96.863, d f=9, P<0.0001$; day 3 pbm, $x^{2}=121.7, d f=12, P<0.0001$; day 6 pbm, $x^{2}=260.1, d f=15$, $P<0.0001$; day $10 \mathrm{pbm}, X^{2}=301.6, d f=9, P<0.0001$. c Morphological forms of $L$. donovani in infected sand flies. The guts of infected females were sampled at 2, 3, 6 and 10 days pbm and parasite morphometry determined as described in methods. The percentage of each form found in infected flies at each time point is shown. Differences among lines were most significant during early infections; day $2 \mathrm{pbm}, X^{2}=190.7, d f=9$, $P<0.0001$; day 3 pbm, $X^{2}=196.5, d f=9, P<0.0001$; day 6 pbm, $X^{2}=8.572, d f=2, P=0.014$; day 10 pbm, $X^{2}=6.755, d f=2, P=0.034$. Abbreviations: E.SP., endoperitrophic space, AMG, abdominal midgut; TMG, thoracic midgut; SV, stomodeal valve

Leishmania are destructed by products of blood meal digestion, like haem and/or reactive oxygen radical generated by free $\mathrm{Fe}^{2+}$, but not directly by midgut proteases as was postulated before [14-16]. Leishmania parasites can profit from inhibition or delay of midgut proteolytic activities by soybean trypsin inhibitor, as was shown in previous studies $[14,16,27]$, because it prolongs the parasites hospitable environment without toxic products of blood meal digestion. It was also observed that sand fly midguts without blood meal have no negative effect on Leishmania in medium. In the study of Doehl et al. [28], promastigotes with added midgut homogenates dissected 12 days pbm grew similarly to negative controls.

Previous studies showed that $P$. argentipes and $P$. orientalis are similarly susceptible to experimental infection with Ethiopian strain of $L$. donovani and even one or two Leishmania promastigotes are sufficient for the establishment of mature late-stage infection [12, 18]. In this study, both natural vectors of $L$. donovani also provided excellent conditions for the development of $L$. donovani parasites after infection by amastigotes, but the kinetics of infections substantially differed. The 
complete development of $L$. donovani in $P$. argentipes was extremely fast; parasites escaped from the endoperitrophic space by day $1 \mathrm{pbm}$, reached the cardia region by day $2 \mathrm{pbm}$ and colonized the stomodeal valve by day $4 \mathrm{pbm}$. In contrast, in $P$. orientalis, Leishmania reached all these aforementioned midgut regions with two-day delay. This striking difference is caused by a very fast blood meal digestion in $P$. argentipes females in comparison with $P$. orientalis. It was recently shown that the peak of proteolytic activities, PM degradation and defecation of blood meal remnants came one to 3 days earlier in $P$. argentipes than $P$. orientalis [12].

As expected, in refractory species $P$. papatasi and $S$. schwetzi, L. donovani did not establish late stage infections. However, importantly, L. donovani amastigotes transformed to promastigotes which developed and multiplied in sand fly midguts until defecation.

High or even $100 \%$ mortality of promastigotes exposed to digested midguts of $P$. argentipes and $P$. orientalis midguts in vitro contradicts successful $L$. donovani development in both natural vectors. One must consider specific conditions accompanying blood digestion in hematophagous insects, where haem generated from haemoglobin catabolism is bound to the peritrophic matrix, which provides its detoxification and protects Leishmania against haem toxicity [29]. In addition, in natural conditions promastigotes escape into the ectoperitrophic space and colonise the abdominal midgut of sand flies at time corresponding to periods when the killing effect on Leishmania in vitro was highest (48 and $72 \mathrm{~h}$ $\mathrm{pbm})$; therefore, they escaped from direct contact with toxic radicals released at the end of blood meal digestion.

Refractoriness of $P$. papatasi to $L$. donovani has been attributed to two different mechanisms. The first hypothesis has suggested that intestinal trypsin-like activity itself prevents development of $L$. donovani in P. papatasi $[14,16]$. The second hypothesis stressed that the vectoral competence in $P$. papatasi correlates with the ability of promastigotes to attach to the sand fly gut [30] and LPG-mediated binding of promastigotes to galectin on midgut epithelium was proposed as the key factor restricting susceptibility of $P$. papatasi to $L$. major [30, 31] and $L$. turanica $[32,33]$. Our results support the second hypothesis as $L$. donovani parasites survived the peak of activity of digestive enzymes, and were able to escape from the PM and colonize the abdominal midgut. Loss of parasites came up later and coincided with the passage of the blood remnants.

\section{Conclusions}

We showed that transition forms of Leishmania are less susceptible to killing effect of semi-digested blood meal (24 $\mathrm{h} \mathrm{pbm})$ than promastigotes and we assume that sand fly midgut proteases do not play important roles in refractoriness of $P$. papatasi to $L$. donovani. The mortality of parasites observed in vitro was unspecific, occurs in natural parasite-vector pairs as well in refractory combinations and increased with the degree of digested blood. In vivo, viable $L$. donovani promastigotes survived and multiplied in $P$. papatasi until the moment of defecation, which confirms insufficiency of $L$. donovani to bind to P. papatasi midgut [30]. Midgut binding, not midgut proteases, are therefore responsible for refractoriness of $P$. papatasi to $L$. donovani.

\section{Additional file}

Additional file 1: Figure S1. Gating strategy. a Gating on single GFP+ cells on GFP-A/GFP-H dotplot. b Gating on Leishmania cells on FSC-A/SSC-A dotplot. c Analysis of DAPI fluorescence on histogram - enumeration of \% DAPI+ (i.e. dead) Leishmania cells. Figure S2. Different Leishmania donovani stages were incubated with midguts of $P$. argentipes dissected at $24 \mathrm{~h}$ postblood meal. Analysis of dead Leishmania was performed using flow cytometry. Percentage of dead cells was assessed on histogram of DAPI fluorescence on gated single GFP-positive Leishmania. Figure S3. Different Leishmania donovani stages were incubated with midguts of $P$. orientalis dissected at $24 \mathrm{~h}$ post-blood meal. Analysis of dead Leishmania was performed using flow cytometry. Percentage of dead cells was assessed on histogram of DAPI fluorescence on gated single GFP-positive Leishmania. Figure S4. Different Leishmania donovani stages were incubated with midguts of P. papatasi dissected at $24 \mathrm{~h}$ post-blood meal. Analysis of dead Leishmania was performed using flow cytometry. Percentage of dead cells was assessed on histogram of DAPI fluorescence on gated single GFPpositive Leishmania. Figure S5. Different Leishmania donovani stages were incubated with midguts of S. schwetzi dissected at $24 \mathrm{~h}$ post-blood meal. Analysis of dead Leishmania was performed using flow cytometry. Percentage of dead cells was assessed on histogram of DAPI fluorescence on gated single GFP-positive Leishmania. Figure S6. Negative control; different Leishmania donovani stages were incubated with saline. Analysis of dead Leishmania was performed using flow cytometry. Percentage of dead cells was assessed on histogram of DAPI fluorescence on gated single GFPpositive Leishmania. Figure S7. Positive control; parasites killed by $1 \%$ formaldehyde and permeabilised by $0.5 \%$ Triton X-100. Percentage of dead cells was assessed on histogram of DAPI fluorescence on gated single GFPpositive Leishmania. (PPTX $346 \mathrm{~kb}$ )

\section{Abbreviations}

AMG: Abdominal midgut; CL: Cutaneous leishmaniasis; DAPI: 4',6-Diamidine-2'phenylindole dihydrochloride; EN: Elongated nectomonads; GFP: Green fluorescence protein; M-CSF: Macrophage colony stimulating factor; MP: Metacyclic promastigotes; pbm: Post-blood meal; PM: Peritrophic matrix; PP: Procyclic promastigotes; RFP: Red fluorescence protein; SP: Short promastigotes; SV: Stomodeal valve; TMG: Thoracic midgut; VL: Visceral leishmaniasis

\section{Acknowledgments}

Not applicable.

Funding

This research was funded by Czech Science Foundation (GACR 13-07 500S) and supported by UNCE (University Research Centre) 204017/2012.

Availability of data and materials

All data generated or analysed during this study are included in this published article.

Authors' contributions

KP designed the study, carried out the experiments, contributed to data analysis and wrote the manuscript. JS designed the study and performed the 
experiments, carried out the statistical analysis and revised the manuscript. JM performed the experiments. TL performed the experiments and revised the manuscript. JJ analysed death cells using flow cytometry. PV designed the study and revised the manuscript. All authors read and approved the final manuscript.

\section{Ethics approval}

Animals used for maintenance of sand flies colonies were maintained and handled in the animal facility of Charles University in Prague in accordance with institutional guidelines and Czech legislation (Act No. 246/1992 and 359/2012 coll. on Protection of Animals against Cruelty in present statutes at large), which complies with all relevant EU guidelines for experimental animals. All experiments were approved by the Committee on the Ethics of Laboratory Experiments of the Charles University in Prague and were performed under the Certificate of Competency (Registration Number: CZ 03069).

\section{Consent for publication}

Not applicable.

\section{Competing interests}

The authors declare that they have no competing interests.

\section{Publisher's Note}

Springer Nature remains neutral with regard to jurisdictional claims in published maps and institutional affiliations.

\section{Author details Czech Republic. \\ Received: 23 June 2017 Accepted: 2 January 2018 \\ Published online: 15 January 2018}

'Department of Parasitology, Faculty of Science, Charles University, Prague, Czech Republic. ${ }^{2}$ Cytometry, Faculty of Science, Charles University, Prague,

\section{References}

1. Dostalova A, Volf P. Leishmania development in sand flies: parasite-vector interactions overview. Parasit Vectors. 2012;5:276.

2. Kamhawi S. Phlebotomine sand flies and Leishmania parasites: friends or foes? Trends Parasitol. 2006;22:439-45

3. Elnaiem DEA. Ecology and control of the sand fly vectors of Leishmania donovani in East Africa, with special emphasis on Phlebotomus orientalis. J Vector Ecol. 2011;36:S23-31.

4. Maroli M, Feliciangeli MD, Bichaud L, Charrel RN, Gradoni L. Phlebotomine sandflies and the spreading of leishmaniases and other diseases of public health concern. Med Vet Entomol. 2013;27:123-47.

5. Ready PD. Biology of phlebotomine sand flies as vectors of disease agents Annu Rev Entomol. 2013:58:227-50.

6. Volf P, Myskova J. Sand flies and Leishmania: specific versus permissive vectors. Trends Parasitol. 2007;23:91-2

7. Dillon RJ, Ivens AC, Churcher C, Holroyd N, Quail MA, Rogers ME, et al. Analysis of ESTs from Lutzomyia longipalpis sand flies and their contribution toward understanding the insect-parasite relationship. Genomics. 2006;88:831-40.

8. Ramalho-Ortigao M, Jochim RC, Anderson JM, Lawyer PG, Pham VM, Kamhawi S, Valenzuela JG. Exploring the midgut transcriptome of Phlebotomus papatasi: comparative analysis of expression profiles of sugar-fed, blood-fed and Leishmania major-infected sandflies. BMC Genomics. 2007;8:300.

9. Jochim RC, Teixeira CR, Laughinghouse A, JB M, Oliveira F, Gomes RB, et al. The midgut transcriptome of Lutzomyia longipalpis: comparative analysis of cDNA libraries from sugar-fed, blood-fed, post-digested and Leishmania infantum chagasi-infected sand flies. BMC Genomics. 2008;9:15.

10. Dillon RJ, Lane RP. Bloodmeal digestion in the midgut of Phlebotomus papatasi and Phlebotomus langeroni. Med Vet Entomol. 1993;7:225-32.

11. Telleria EL, de Araujo APO, Secundino NF, d'Avila-Levy CM, Traub-Cseko YM. Trypsin like serine proteases in Lutzomyia longipalpis - expression, activity and possible modulation by Leishmania infantum chagasi. PLoS One. 2010;5:e10697.

12. Pruzinova K, Sadlova J, Seblova V, Homola M, Votypka J, Volf P. Comparison of bloodmeal digestion and the peritrophic matrix in four sand fly species differing in susceptibility to Leishmania donovani. PLoS One. 2015;10:e0128203.

13. Pruzinova K, Votypka J, Volf $P$. The effect of avian blood on Leishmania development in Phlebotomus duboscai. Parasit Vectors. 2013;6:254.
14. Borovsky D, Schlein Y. Trypsin and chymotrypsin-like enzymes of the sandfly Phlebotomus papatasi infected with Leishmania and their possible role in vector competence. Med Vet Entomol. 1987;1:235-42.

15. Pimenta PFP, Modi GB, Pereira ST, Shahabuddin M, Sacks DL. Novel role for the peritrophic matrix in protecting Leishmania from the hydrolytic activities of the sand fly midgut. Parasitology. 1997;115:359-69.

16. Schlein Y, Jacobson RL. Resistance of Phlebotomus papatasi to infection with Leishmania donovani is modulated by components of the infective bloodmeal. Parasitology. 1998;117:467-73.

17. Schlein Y, Romano H. Leishmania major and Leishmania donovani - effects on proteolytic enzymes of Phlebotomus papatasi (Diptera, Psychodidae). Exp Parasitol. 1986;62:376-80.

18. Seblova V, Volfova V, Dvorak V, Pruzinova K, Votypka J, Kassahun A, et al. Phlebotomus orientalis sand flies from two geographically distant Ethiopian localities: biology, genetic analyses and susceptibility to Leishmania donovani. PLoS Negl Trop Dis. 2013;7:e2187.

19. Hoogstraal H, Heyneman D. Leishmaniasis in Sudan Republic. 30. Final epidemiologic report. Am J Trop Med Hyg. 1969;18:1091-210.

20. Sadlova J, Dvorak V, Seblova V, Warburg A, Votypka J, Volf P. Sergentomyia schwetzi is not a competent vector for Leishmania donovani and other Leishmania species pathogenic to humans. Parasit Vectors. 2013;6:186.

21. Volf P, Volfova V. Establishment and maintenance of sand fly colonies. J Vector Ecol. 2011;36:S1-9.

22. Chang KP. Human cutaneous Leishmania in a mouse macrophage line: propagation and isolation of intracellular parasites. Science. 1980;209:12402.

23. Myskova J, Votypka J, Volf P. Leishmania in sand flies: comparison of quantitative polymerase chain reaction with other techniques to determine the intensity of infection. J Med Entomol. 2008;45:133-8.

24. Sadlova J, Myskova J, Lestinova T, Votypka J, Yeo M, Volf P. Leishmania donovani development in Phlebotomus argentipes: comparison of promastigote- and amastigote-initiated infections. Parasitology. 2017;144:403-10.

25. Schlein Y, Jacobson RL. Hemoglobin inhibits the development of infective promastigotes and chitinase secretion in Leishmania major cultures. Parasitology. 1994;109:23-8.

26. Balla J, Vercellotti GM, Jeney V, Yachie A, Varga Z, Eaton JW, Balla G. Heme, heme oxygenase and ferritin in vascular endothelial cell injury. Mol Nutr Food Res. 2005:49:1030-43.

27. Dillon RJ, Lane RP. Influence of Leishmania infection on blood meal digestion in the sandflies Phlebotomus papatasi and Phlebotomus langeronia. Parasitol Res. 1993;79:492-6.

28. Doehl JS, Sadlova J, Aslan H, Pruzinova K, Metangmo S, Votypka J, et al. Leishmania HASP and SHERP genes are required for in vivo differentiation, parasite transmission and virulence attenuation in the host. PLoS Pathog. 2017; 13:e1006130

29. Pascoa V, Oliveira PL, Dansa-Petretski M, Silva JR, Alvarenga PH, JacobsLorena M, Lemos FJ. Aedes aegypti peritrophic matrix and its interaction with heme during blood digestion. Insect Biochem Molec. 2002;32:517-23.

30. Pimenta PFP, Saraiva EMB, Rowton E, Modi GB, Garraway LA, Beverley SM, et al. Evidence that the vectorial competence of phlebotomine sand flies for different species of Leishmania s.s. controlled by structural polymorphisms in the surface lipophosphoglycan. Proc Natl Acad Sci USA. 1994;91:9155-9.

31. Kamhawi S, Ramalho-Ortigao M, Pham VM, Kumar S, Lawyer PG, Turco SJ, et al. A role for insect galectins in parasite survival. Cell. 2004;119:329-41.

32. Chajbullinova A, Votypka J, Sadlova J, Kvapilova K, Seblova V, Kreisinger J, et al. The development of Leishmania turanica in sand flies and competition with L. major. Parasit Vectors. 2012;5:219.

33. Volf P, Nogueira PM, Myskova J, Turco SJ, Soares RP. Structural comparison of lipophosphoglycan from Leishmania turanica and L. major, two species transmitted by Phlebotomus papatasi. Parasitol Int. 2014;63:683-6. 